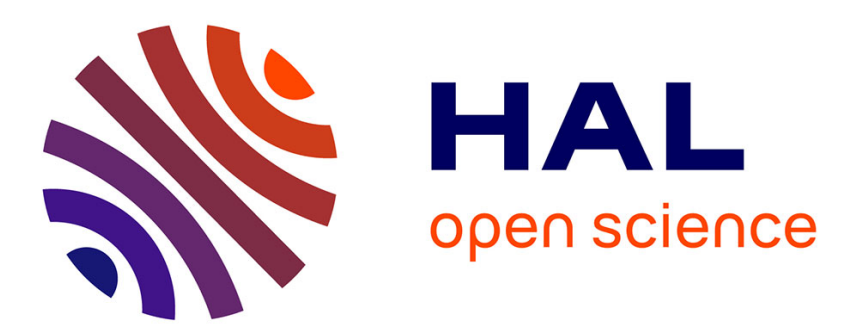

\title{
Sur de nouveaux matériaux particulaires pour l'enregistrement magnétique dérivés du nitrure de fer $\mathrm{Fe} 4 \mathrm{~N}$
}

Samir F. Matar, Gérard Demazeau

\section{- To cite this version:}

Samir F. Matar, Gérard Demazeau. Sur de nouveaux matériaux particulaires pour l'enregistrement magnétique dérivés du nitrure de fer Fe4N. Journal de Physique IV Proceedings, 1992, 02 (C3), pp.C317-C3-22. 10.1051/jp4:1992302 . jpa-00251507

\section{HAL Id: jpa-00251507 https://hal.science/jpa-00251507}

Submitted on 1 Jan 1992

HAL is a multi-disciplinary open access archive for the deposit and dissemination of scientific research documents, whether they are published or not. The documents may come from teaching and research institutions in France or abroad, or from public or private research centers.
L'archive ouverte pluridisciplinaire HAL, est destinée au dépôt et à la diffusion de documents scientifiques de niveau recherche, publiés ou non, émanant des établissements d'enseignement et de recherche français ou étrangers, des laboratoires publics ou privés. 


\section{Sur de nouveaux matériaux particulaires pour l'enregistrement magnétique dérivés du nitrure de fer $\mathrm{Fe}_{4} \mathrm{~N}$}

\section{S. MATAR et G. DEMAZEAU}

Laboratoire de Chimie du Solide du C.N.R.S. et Interface Hautes Pressions LCS-ENSCPB, Université Bordeaux 1, 33405 Talence, France

Résumé. La coercivité du matériau particulaire Fean destiné à l'enregistrement magnétique a été améliorée soit par des substitutions dans le coeur ou par des enrobages de la surface. Les meilleurs résultats sont obtenus lorsque la couche de surface peut jouer un rôle magnétique au travers d'interactions d'échange entre les atomes des couches supérieures de la particule et ceux de la surface.

Abstract. The coercivity of the particulate material Fean aimed at magnetic recording has been improved throughout substitutions in the bulk or surface coatings. The best results are obtained when the surface layer can play a magnetic role through exchange interactions with the iron atoms of the crystalitites.

1. Introduction.

L'enregistrement magnétique de haute densité requiert des valeurs élevées tant pour le champ coercitif que pour l'aimantation à saturation $\left(700 \leq H_{c} \leq 1000\right.$ oe avec $\sigma$ s supérieur a $100 \mathrm{uem} / \mathrm{g}_{\text {.). }}$. Du fait de leur forte aimantation à saturation (de l'ordre de 180 uem/g.), les particules dérivées du fer constituent un pigment magnétique très prometteur. Cependant étant donné que la taille du monodomaine magnétique est faible, l'obtention de valeurs importantes de $\mathrm{Hc}$ implique des particules très petites. Un tel état de division conduit à des surfaces spécifiques très développées facilitant ainsi la dégradation chimique de telles particules par oxydation soit par l'oxygène de l'air, soit par la vapeur d'eau. Afin d'entraver ce phénomène deux voies de recherche semblaient intéressantes : i) soit l'élaboration de nouveaux matériaux ferro- ou ferrimagnétiques présentant une valeur de $\sigma_{s}$ du même 
ordre de grandeur que celle du fer - et dans ce cas nos efforts se sont portés sur Fe4N $\left(\sigma_{s_{300 x}} \cong 200 \mathrm{uem} / g \cdot{ }[1]_{g} i i\right)$ soit la passivation chimique de surface par enrobage ( des essais ont été réalisés notamment sur FeđN ) afin d'accroftre sa stabilité chimique.

2. Amélioration des propriêtés magnétiques de FeđNㅡㅁ par enrobages et substitutions.

L'obtention de telles particules magnétiques s'effectuant par un processus de réaction thermique de type solide -gaz a partir de précurseurs (sel ou oxyde de fer), dans un flux gazeux nitrurant, une attention particulière a été portée sur la préparation de ceux-ci. La morphologie aciculaire a été sélectionnée, l'application dans le domaine d'enregistrement longitudinal étant ciblée. L'oxalate de fer $\left(\mathrm{FeC}_{2} \mathrm{O}_{4}, 2 \mathrm{H}_{2} \mathrm{O}\right)$ et la goethite (FeOOH) ont eté choisis comme precurseurs. La décomposition de ces derniers s'effectue sous un débit gazeux ammoniac-hydrogène (nitrurant-réducteur) à $400^{\circ} \mathrm{C}$ durant 5 heures dans un réacteur de type lit fluidisé. Alors que les caractéristiques magnétiques intrinsèques des particules de FeđN elaborées a partir de tels réactifs sont relativement élevées ( $\sigma_{s} \cong 170$ uem/g), les propriétés extrinsèques : Hc et

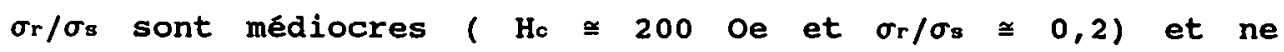
permettent pas d'envisager une utilisation potentielle pour l'enregistrement. L'analyse par microscopie electronique à balayage et en transmission (MEB et MET) des pigments obtenus a montré que ces particules conservent la forme générale de celles du précurseur (transformation pseudo-isomorphique). L'examen des clichés MET précise l'état de division, les particules magnétiques etant formées de microcristallites sphérolides non-jointives. La maitrise du processus de subdivision lors de la transformation précurseur $\rightarrow$ Fe4N s'est avérée nécessaire afin de controler les valeurs de or et $\mathrm{Hc}$.

i- Etude de la subdivision des particules aciculaires de précurseur lors de l'utilisation d'enrobages.

Un traitement de surface des particules du précurseur était susceptible de réduire les phénomènes de frittage 
(évitant la formation de particules polydomaines magnétiques) d'une part et de préserver la particule dans une forme pas trop divisée (grains à comportement superparamagnétique) d'autre part a été entrepris. Les initiateurs d'enrobants ont été choisis parmi l'acide phosphorique, l'hydrogénophosphate d'ammonium ( $\left.\mathrm{NH}_{4} \mathrm{H}_{2} \mathrm{PO}_{4}\right)$ ou $\mathrm{l}^{\prime}$ acide borique (H3BO3). Les particules magnétiques obtenues après réaction présentent un accroissement du champ coercitif : Hc $\cong 450$ Oe. Une analyse par spectroscopie Auger de celles-ci a montré que l'enrobant ( ions borate ou phosphate) se place préférentiellement en surface.

ii-Contrôle de la subdivision des particules aciculaires de précurseur à l'aide de substituants appropriés.

La présence de deux sites cristallographiques dans Fe4N permettait, au travers de la modification des énergies de surface concernées par chacun des sites d'envisager un contrôle du processus de croissance par des substitutions selectives. Selon la taille de l'ion substituant il était possible d'envisager une substitution préférentielle sur l'un des sites : i.e. dans Fe(I) ${ }_{1-x^{M}} \mathrm{Fe}(\mathrm{II})_{3} \mathrm{~N}$ la substitution de $\mathrm{M}$ se faisant préférentiellement sur le site $\mathrm{Fe}(\mathrm{I})$ (Fig. 1). Tel est le cas de $S n$ [2], en revanche $M n$ se substitue indifféremment sur les deux sites du fer [3]. De telles substitutions spécifiques peuvent modifier le processus de transformation : particule de précurseur $\rightarrow$ particule magnétique en réduisant la taille du grain magnétique. Les meilleures performances sont ainsi obtenues pour un taux de substitution voisin de $x=0,5$ en $\mathrm{Sn}$ (ou $\mathrm{Mn}$ ) pour lequel $\mathrm{Hc} \cong 600$ Oe avec des particules ayant un comportement de monodomaines magnetiquee $, 1 \cong n, 15 n \mathrm{~nm}, 1$, tail10 moyenne du grain).

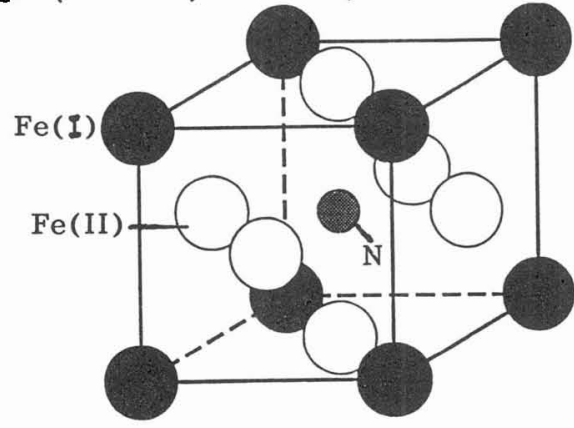

Fig. 1.- Structure cristalline de FeaN. 
3. Passivation de surface des pigments magnétiques.

Quels que soient les précurseurs et les conditions de préparation utilisées lors de l'élaboration des pigments à base de Fe4N, il apparait difficile d'éviter la formation d'oxyde de fer- $\alpha$ antiferromagnétique à la surface des grains magnétiques du fait de leur dégradation chimique au contact de l'oxygène de l'air. En outre un tel phénomène implique une diminution de l'aimantation. Nous avons donc etudié la formation d'une couche passivante d'oxyde mixte et son influence sur les propriétés magnétiques en résultant. Afin d'une part de limiter la quantité de fer ainsi mobilisée dans la couche superficielle et d'autre part de faciliter une meilleure adhérence de entre celle-ci et la particule magnétique, la perovskite LnFeO3, oxyde de structure proche de FesN et contenant du fer a été choisie. La terre rare peut en outre -en fonction de sa structure électronique- induire une anisotropie magnétocristalline. Partant de la goethite de morphologie aciculaire comme précurseur, une étude a été effectuée en utilisant le néodyme. Compte tenu de la structure électronique anisotrope de $\mathrm{Nd}^{\mathrm{III}}$ et afin de déterminer le rôle du substituant dans la couche de surface, une étude comparative a été menée avec $\mathrm{La}^{\mathrm{III}}$ présentant une configuration électronique isotrope. Le précurseur d'enrobant (l'oxalate de néodyme précipité à partir d'une solution aqueuse du sel correspondant) est déposé à la surface des particules de FeOOH. Les cristallites ainsi enrobées sont ensuite soumises à un traitement thermique en réacteur à lit fluidisé $\left(1<\mathrm{NH}_{3} / \mathrm{H}_{2}<2\right.$, $t_{1}=1 \mathrm{~h}$. à $130^{\circ} \mathrm{C}$ puis $t_{2}=6 \mathrm{~h}$. à $\left(400<\mathrm{T}<450^{\circ} \mathrm{C}\right)$. L'analyse radiocristallographique souligne qu'en plus du spectre caractéristique de Fe4N apparaissent des raies attribuables à une phase de type LnFeO3. La largeur des raies de diffraction de cette phase permet de suggérer que la phase de surface est mal cristallisée. La composition approximative de celle-ci ainsi que sa localisation ont été montrées par spectroscopie Auger. Les clichés de microscopie électronique ne permettent pas de mettre en évidence de modifications de la taille des grains magnétiques. En revanche une meilleure texture des 
particules est observée avec l'augmentation du taux de terre rare [4]. Une étude de la variation de la surface spécifique en fonction de la composition croissante de terre rare a permis de mettre en evidence sa diminution.

Les phases de formulation LnFeO3 ( $\mathrm{Ln}=\mathrm{La}, \mathrm{Nd})$ sont caractérisées par un ordre antiferromagnétique (TN $\cong 700 \mathrm{~K}$ ). La maille pseudo-cubique de ces phases a un paramètre $a_{0}=3,876 \AA$ $(\mathrm{Ln}=\mathrm{Nd})$ proche de celui de Fe4N $(3,797 \AA)$ ce qui peut faciliter une croissance épitaxiale de la couche de surface.

Les variations du champ coercitif et de l'aimantation a saturation pour $\mathrm{Ln}=\mathrm{La}(2 \mathrm{a})$ et $\mathrm{Nd}(2 \mathrm{~b})$ sont données à $l \mathrm{a}$ figure 2 . Alors qu'une diminution régulière de $\sigma_{s}$ avec le taux de terre rare en surface est observée, on note une variation plus complexe de Hc.

La diminution de $\sigma_{s}$ avec le taux de in pourrait être interprétée par la mobilisation d'un nombre croissant d'atomes de fer du coeur de la particule pour former $\mathrm{LnFeO}_{3}$ en surface. La variation de $H c$ présentant deux maxima - l'un pour les faibles taux en In (2,5 \%) l'autre vers $\cong 10 \%-$, elle a été déconvoluée en deux courbes. La première a un maximum à $\mathrm{Hc}$ $\cong 550$ oe pour $\mathrm{Ln}=\mathrm{La}$ et $\mathrm{Hc} \cong 750$ oe pour $\mathrm{Ln}=\mathrm{Nd}$ (la différence pouvant être attribuée à la configuration électronique anisotrope de $\mathrm{Nd}^{\mathrm{III}}$ ). Ce phénomène suggère une interaction magnétique d'échange à la surface entre les atomes de fer de $\mathrm{LnFeO}_{3}$ et ceux de Fe4N. Elle devrait être facilitée par les similarités des deux réseaux (épitaxie) et de la configuration antiferro-/ferro- magnétique (AF/F) entre la surface et le volume [4]. La deuxième courbe a un maximum a $\cong$ 10\%. Dans ce cas l'amélioration de la texture et la diminution de la surface spécifique pour les taux élevés en Ln pourrait être induite par une diminution des propriétés liée à l'accroissement de la couche de surface - phénomène pouvant également augmenter l'anisotropie de forme.

4. Conclusions.

Cette étude a permis de montré qu'il est possible d'améliorer la coercivité de matériaux magnétiques 
particulaires derives de Fe4N par voies de substitutions dans le coeur ou d'enrobage de la surface. Les meilleurs résultats sont obtenus lorsque la couche de surface peut jouer un role magnétique au travers d'interactions d'échange avec les atomes magnetiques des microcristallites.

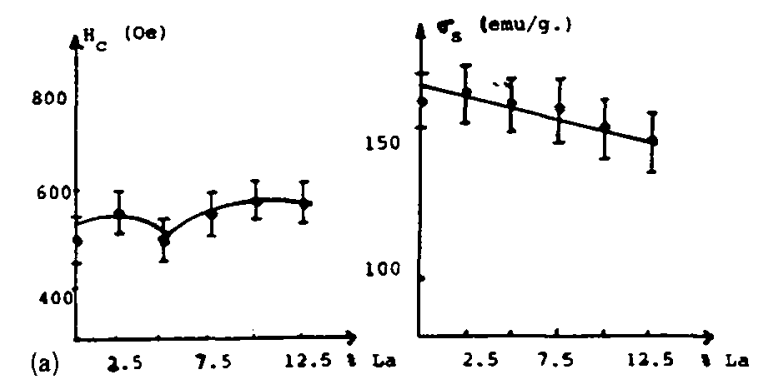

(b)

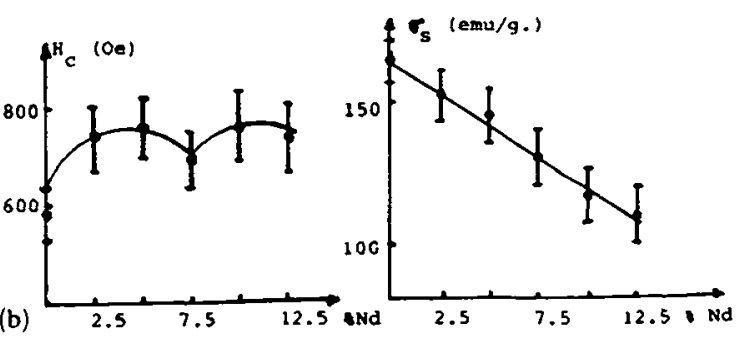

Fig. 2.- Variations du champ coercitif et de l'aimantation en fonction du taux de La (a) et de $\mathrm{Nd}(\mathrm{b})$ à la surface de Fe4N.

\section{Bibliographie.}

1. G. Demazeau, D. Andriamandroso, M. Pouchard, B. Tanguy et P. Hagenmuller. C.R. Acad. Sci. 2, 297, 843 (1983).

2. D. Andriamandroso, I. Fefilatiev, G. Demazeau, L. Fournès et M. Pouchard. Mater. Res. Bull. 19, 11897 (1984).

3. B. Siberchicot, S.F. Matar, L. Fournès, G. Demazeau et P. Hagenmuller. J. Solid St. Chem., 84, 10 (1989).

4. S. Berthon, S. Matar, B. Siberchicot et G. Demazeau. Mater. Sc. and Engin., B12, 383 (1992). 